\title{
Octanuclear zinc(II) and cobalt(II) clusters produced by cooperative tetrameric assembling of oxime chelate ligands
}

\author{
Shigehisa Akine, Wenkui Dong, and Tatsuya Nabeshima* \\ Department of Chemistry, University of Tsukuba, Tsukuba, Ibaraki 305-8571, Japan \\ Email: nabesima@chem.tsukuba.ac.jp
}

Supporting Information 


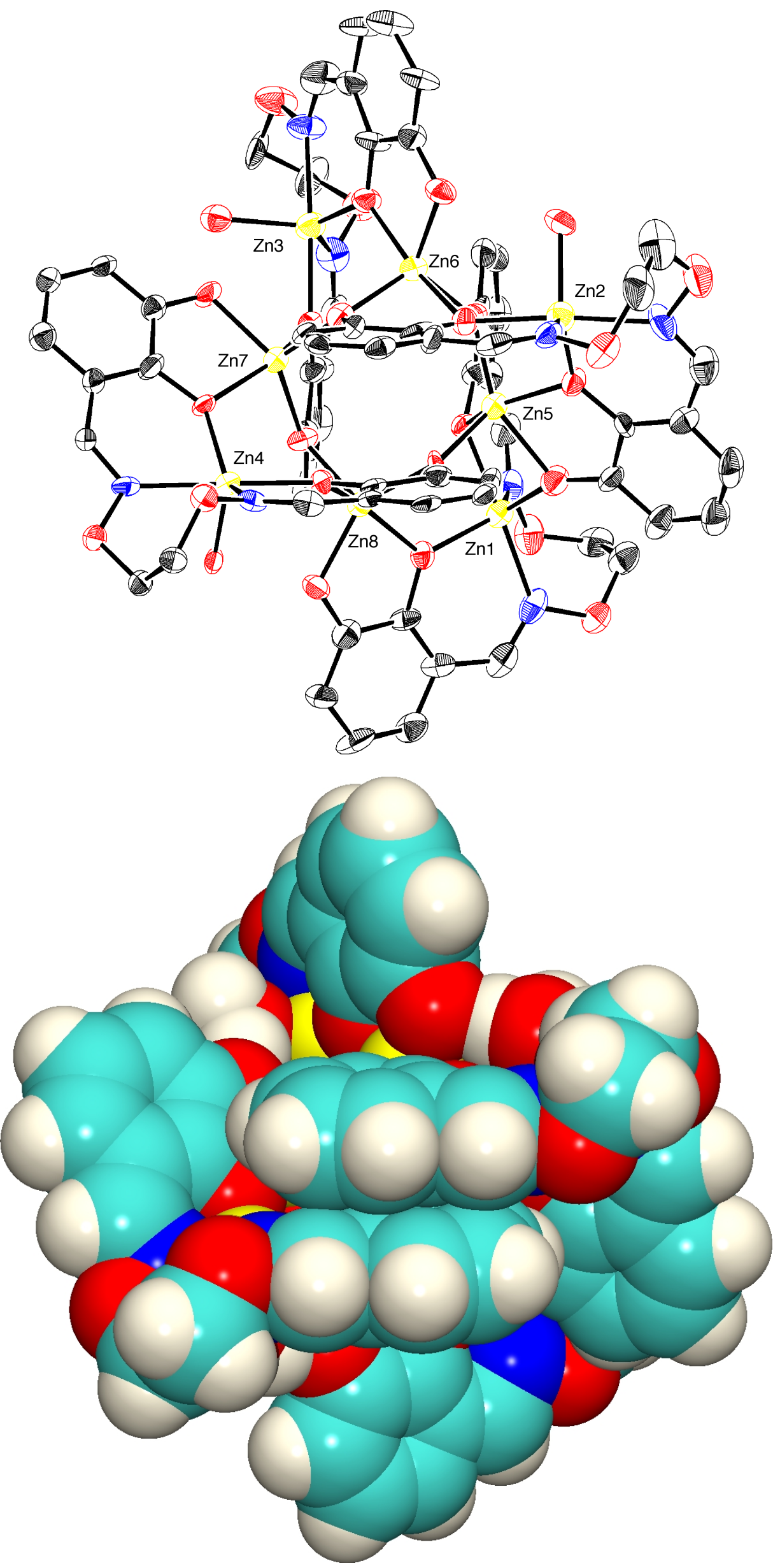

Figure S1. Crystal structure of $\left[\mathrm{L}_{4} \mathrm{Zn}_{8}\left(\mathrm{H}_{2} \mathrm{O}\right)_{3}\right]$ (Molecule 1). 

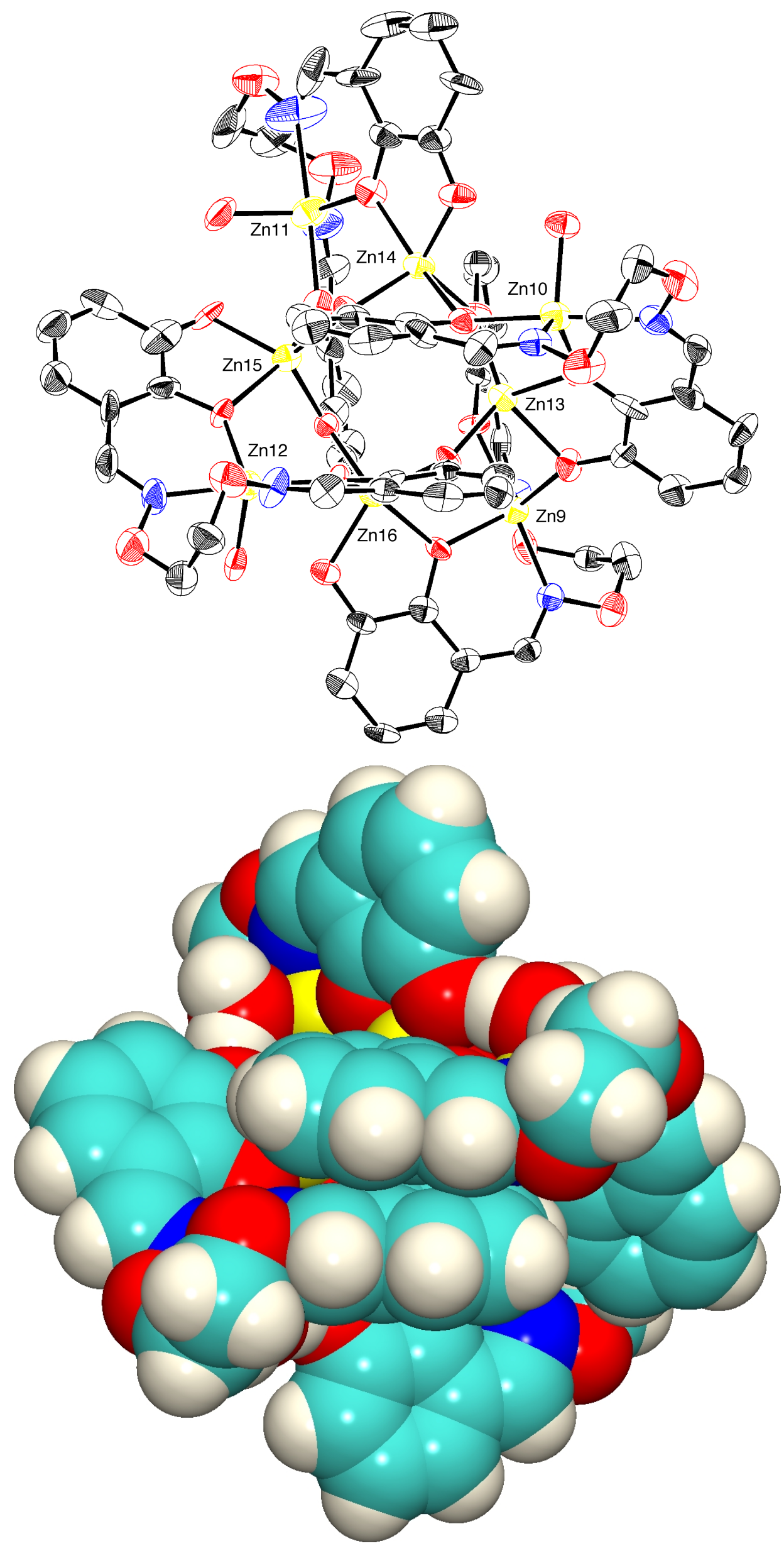

Figure S2. Crystal structure of $\left[\mathrm{L}_{4} \mathrm{Zn}_{8}\left(\mathrm{H}_{2} \mathrm{O}\right)_{3}\right]$ (Molecule 2). 
Table S1. Selected interatomic distances $(\AA)$ in $\left[\mathrm{L}_{4} \mathrm{Zn}_{8}\left(\mathrm{H}_{2} \mathrm{O}\right)_{3}\right]$ and $\left[\mathrm{L}_{4} \mathrm{Co}_{8}\left(\mathrm{H}_{2} \mathrm{O}\right)_{2} \mathrm{X}\right]\left(\mathrm{X}=\mathrm{H}_{2} \mathrm{O}\right.$ or $\mathrm{EtOH})$.

\begin{tabular}{|c|c|c|c|c|c|}
\hline \multicolumn{2}{|c|}{$\begin{array}{l}{\left[\mathrm{L}_{4} \mathrm{Zn}_{8}\left(\mathrm{H}_{2} \mathrm{O}\right)_{3}\right]} \\
\text { molecule } 1\end{array}$} & \multicolumn{2}{|l|}{ molecule 2} & \multicolumn{2}{|c|}{$\begin{array}{l}{\left[\mathrm{L}_{4} \mathrm{Co}_{8}\left(\mathrm{H}_{2} \mathrm{O}\right)_{2} \mathrm{X}\right]} \\
\left(\mathrm{X}=\mathrm{H}_{2} \mathrm{O} \text { or } \mathrm{EtOH}\right)\end{array}$} \\
\hline$\overline{\mathrm{Zn} 1-\mathrm{O} 1}$ & $2.019(8)$ & Zn9-O28 & $1.973(8)$ & Co1-O1 & $1.959(4)$ \\
\hline Zn1-O5 & $1.976(9)$ & Zn9-O32 & $2.006(9)$ & Co1-O5 & $2.043(4)$ \\
\hline Zn1-O8 & $1.965(11)$ & Zn9-O35 & $2.012(10)$ & Co1-O8 & $2.012(4)$ \\
\hline Zn1-N1 & $2.095(15)$ & Zn9-N9 & $2.020(12)$ & Co1-N1 & $2.067(5)$ \\
\hline Zn1-N2 & $2.073(15)$ & Zn9-N10 & $1.998(13)$ & Co1-N2 & $2.038(5)$ \\
\hline $\mathrm{Zn2-O7}$ & $1.987(9)$ & Zn10-O34 & $1.959(10)$ & $\mathrm{Co} 2-\mathrm{O} 7$ & $1.970(4)$ \\
\hline $\mathrm{Zn2-O11}$ & $1.995(9)$ & Zn10-O38 & $2.031(10)$ & Co2-O11 & $2.053(4)$ \\
\hline $\mathrm{Zn} 2-\mathrm{O} 25$ & $2.035(11)$ & Zn10-O52 & $1.988(10)$ & $\mathrm{Co} 2-\mathrm{O} 25$ & $1.994(5)$ \\
\hline $\mathrm{Zn} 2-\mathrm{N} 3$ & $2.115(14)$ & Zn10-N11 & $2.179(15)$ & Co2-N3 & $2.153(6)$ \\
\hline Zn2-N4 & $1.988(13)$ & Zn10-N12 & $1.966(14)$ & Co2-N4 & $2.000(6)$ \\
\hline Zn3-O13 & $1.945(9)$ & Zn11-O40 & $1.959(11)$ & Co3-O13 & $1.957(4)$ \\
\hline Zn3-O17 & $2.077(9)$ & Zn11-O44 & $2.016(11)$ & Co3-O17 & $2.035(4)$ \\
\hline $\mathrm{Zn3-O26}$ & $1.988(10)$ & Zn11-O53 & $1.971(10)$ & $\mathrm{Co} 3-\mathrm{O} 26$ & $2.016(5)$ \\
\hline Zn3-N5 & $2.136(13)$ & Zn11-N13 & $2.14(2)$ & Co3-N5 & $2.166(6)$ \\
\hline Zn3-N6 & $2.011(15)$ & Zn11-N14 & $1.931(18)$ & Co3-N6 & $1.987(6)$ \\
\hline Zn4-O19 & $1.971(8)$ & $\mathrm{Zn} 12-\mathrm{O} 46$ & $1.961(9)$ & Co4-O19 & $1.962(5)$ \\
\hline $\mathrm{Zn} 4-\mathrm{O} 23$ & $1.989(8)$ & $\mathrm{Zn} 12-\mathrm{O} 50$ & $1.977(8)$ & $\mathrm{Co} 4-\mathrm{O} 23$ & $2.018(5)$ \\
\hline $\mathrm{Zn} 4-\mathrm{O} 27$ & $1.989(9)$ & Zn12-O54 & $1.960(12)$ & $\mathrm{Co} 4-\mathrm{O} 27$ & $2.023(6)$ \\
\hline Zn4-N7 & $2.187(11)$ & Zn12-N15 & $2.100(13)$ & Co4-N7 & $2.158(6)$ \\
\hline Zn4-N8 & $2.010(11)$ & Zn12-N16 & $2.033(14)$ & Co4-N8 & $2.022(8)$ \\
\hline Zn5-O5 & $2.074(8)$ & Zn13-O32 & $2.056(9)$ & Co5-O5 & $2.085(4)$ \\
\hline Zn5-O6 & $2.015(10)$ & Zn13-O33 & $2.025(9)$ & Co5-O6 & $2.025(4)$ \\
\hline Zn5-O7 & $1.931(8)$ & Zn13-O34 & $2.007(10)$ & Co5-O7 & $2.043(4)$ \\
\hline Zn5-O8 & $2.096(10)$ & Zn13-O35 & $2.104(10)$ & Co5-O8 & $2.052(4)$ \\
\hline $\mathrm{Zn5-O} 24$ & $1.943(8)$ & Zn13-O51 & $1.919(8)$ & $\mathrm{Co5}-\mathrm{O} 24$ & $1.968(4)$ \\
\hline Zn6-O6 & $1.923(10)$ & Zn14-O33 & $1.912(9)$ & Co6-O6 & $1.954(4)$ \\
\hline Zn6-O11 & $2.222(10)$ & $\mathrm{Zn} 14-\mathrm{O} 38$ & $2.280(10)$ & Co6-O11 & $2.256(4)$ \\
\hline Zn6-O12 & $1.998(9)$ & Zn14-O39 & $1.967(9)$ & Co6-O12 & $1.989(4)$ \\
\hline
\end{tabular}




\begin{tabular}{llllll}
\hline Zn6-O13 & $2.129(10)$ & Zn14-O40 & $2.101(11)$ & Co6-O13 & $2.127(4)$ \\
Zn6-O14 & $1.885(9)$ & Zn14-O41 & $1.926(11)$ & Co6-O14 & $1.946(4)$ \\
Zn7-O12 & $1.927(9)$ & Zn15-O39 & $1.945(9)$ & Co7-O12 & $1.982(4)$ \\
Zn7-O17 & $2.228(9)$ & Zn15-O44 & $2.228(10)$ & Co7-O17 & $2.202(4)$ \\
Zn7-O18 & $1.964(9)$ & Zn15-O45 & $1.934(9)$ & Co7-O18 & $1.977(4)$ \\
Zn7-O19 & $2.140(8)$ & Zn15-O46 & $2.179(9)$ & Co7-O19 & $2.230(5)$ \\
Zn7-O20 & $1.945(9)$ & Zn15-O47 & $1.944(10)$ & Co7-O20 & $1.945(4)$ \\
Zn8-O1 & $2.126(9)$ & Zn16-O28 & $2.095(9)$ & Co8-O1 & $2.163(4)$ \\
Zn8-O2 & $1.937(9)$ & Zn16-O29 & $1.968(9)$ & Co8-O2 & $1.977(4)$ \\
Zn8-O18 & $1.952(9)$ & Zn16-O45 & $1.961(9)$ & Co8-O18 & $1.976(4)$ \\
Zn8-O23 & $2.134(8)$ & Zn16-O50 & $2.126(9)$ & Co8-O23 & $2.193(4)$ \\
Zn8-O24 & $1.961(8)$ & Zn16-O51 & $1.984(9)$ & Co8-O24 & $1.975(4)$ \\
\hline
\end{tabular}

